\title{
Evaluation of a ToF Camera for Remote Surveying of Underground Cavities Excavated by Jet Boring
}

\author{
Carolyn Ingram ${ }^{\mathrm{a}, \mathrm{b}, *}$, Joshua Marshall ${ }^{\mathrm{b}}$ \\ ${ }^{a}$ Cameco Corporation, 2121-11th Street West, Saskatoon, Canada S7M 1J3 \\ ${ }^{b}$ Mining Systems Laboratory, Queen's University, Kingston, Canada K7L 3N6
}

\begin{abstract}
Cigar Lake is a high-grade uranium deposit, located in northern Saskatchewan, Canada. In order to extract the uranium ore remotely, thus ensuring minimal radiation dose to workers and also to access the ore from stable ground, the Jet Boring System (JBS) was developed by Cameco Corporation. This system uses a high-powered water jet to remotely excavate cavities. Survey data is required to determine the final shape, volume, and location of the cavity for mine planning purposes and construction. This paper provides an overview of the challenges involved in remotely surveying a JBS-mined cavity and studies the potential use of a time-of-flight (ToF) camera for remote cavity surveying. It reports on data collected and analyzed from inside an experimental environment as well as on real data acquired on site from the Cigar Lake and Rabbit Lake mines.
\end{abstract}

Keywords: Underground mine construction, Cavity surveying, ToF camera, C-ALS, 3D point clouds, Registration, Segmentation, Jet boring 2010 MSC: 00-01, 99-00

\section{Introduction}

Cigar Lake is the world's second largest high-grade uranium deposit, located in northern Saskatchewan, Canada. A unique mining method was developed,

\footnotetext{
* Corresponding author

Email addresses: carolyn_ingram@cameco.com (Carolyn Ingram), joshua.marshall@queensu.ca (Joshua Marshall)
}

Preprint submitted to Automation in Construction 
called the Jet Boring System (JBS), to access the high-grade uranium orebody, 5 which is situated in water-saturated sandstone. Before mining begins, the orebody and surrounding rock is frozen to strengthen it and also to prevent inflows. A pilot hole is then drilled up through the ore body and cased, providing a path for the jet string and nozzle. The jetting begins at the top of the ore body and progresses downwards in periods, as the jet rotates and traverses about its axis, until the lower limit of the ore body is reached. As the cavity is jetted, the ore slurry falls through the annulus of the pilot hole casing and jet pipe, and into a slurry storage tank before being pumped to the run of mine (ROM) area. From there, further processing of the slurry occurs underground, after which it is finally pumped to surface [1].

15 The purpose of the cavity survey, which will take place between periods of jetting, is to provide feedback to the operator located beneath the ore body (see Figure 11, indicating the dimensions of the cavity. During excavation, it is important to achieve maximum recovery while preventing ground instability that may be caused by too large of a cavity. Following the mine plan, the operator will need to know if it is necessary to focus the jet on a particular area within the cavity, to change the jetting parameters, or to cease mining. Additionally, the final location of the cavity and its dimensions are required in order to update resource estimates and production values. The volume of required backfill can also be determined if the cavity shape is known. Knowledge of the JBS's performance and the ability to optimize procedures to ensure efficient maximum recovery are necessary for a successful mining program. The challenge is to find an appropriate tool that can acquire reliable data within the extreme cavity environment, including fog buildup and flying debris during mining, as well as a space that is wet, dark, and cold.

so This paper considers three devices that could be used for surveying at remote cavities and summarizes the experiments and analyses that have been performed. Two of the three devices that are described, are currently planned to survey the remote cavities at Cigar Lake. These include, firstly, an ultrasonic device and secondly, a commercial laser scanning system. As a potential 


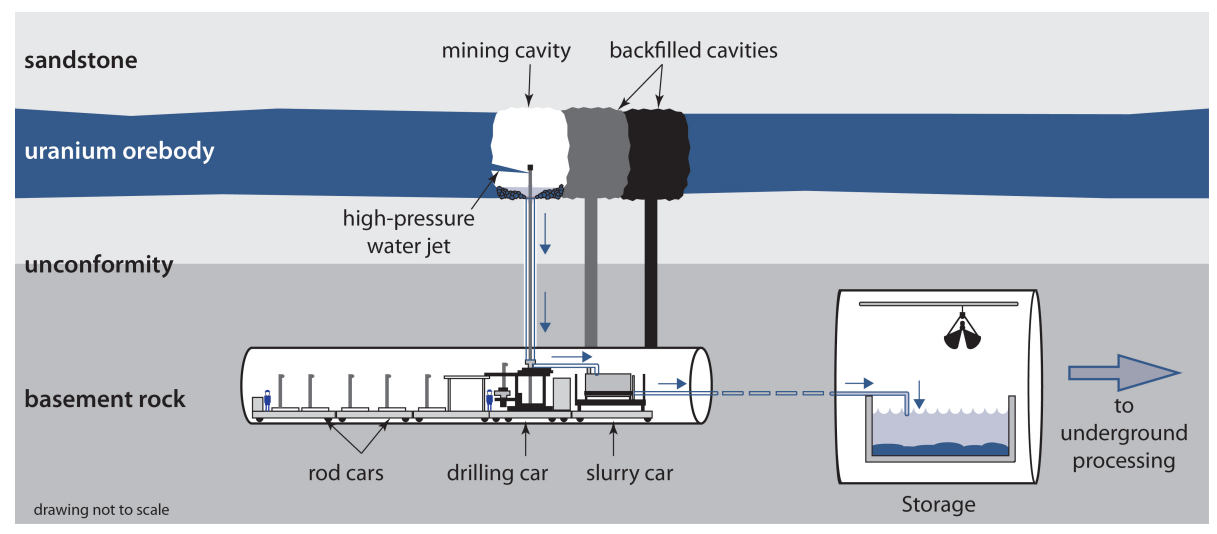

Figure 1: JBS mining schematic for Cigar Lake (image courtesy of Cameco Corporation)

alternative to one or both of the aforementioned devices, the third device, a time-of-flight (ToF) camera, is examined and compared to the 3D laser scanning tool. Experiments and data collection were performed in a test cavity, which was constructed for this project, to isolate and simulate the effects of different environmental conditions expected to be present in the remote cavities.

40 The ToF camera was also taken to the Cigar Lake and Rabbit Lake mine sites to collect data that is representative of what would generally be seen inside a mine.

With the ToF camera, some advanced data analysis techniques may be possible and are examined in this paper for application within remote cavity surveying. Registration [2, 3, 4, 5] can be used to determine the change in position of the camera by calculating the rotation and translation in the point cloud acquired from different viewpoints. It is proposed that this technique be used to supplement and improve upon pose estimated by odometry devices. Segmentation [3, 6, 7] is another analysis technique that is explored for use in identifying the various distinct mineral compositions that may be found inside the remote cavities. In particular, segmentation could be used to define the relative location of ore body extents, giving further information to jet boring operators and providing opportunity for optimized mine planning. 


\section{Remote Cavity Surveying}

This section describes the Cigar Lake application and provides an introduction to the questions surrounding sensor selection.

\subsection{Cigar Lake Application}

At Cigar Lake, the original cavity survey system field testing took place in 2000, from April through November [8]. The first set of tests were conducted in a vertical culvert in an underground raise, with simulated rock conditions similar to those expected during a typical mining situation. The next phase of testing took place in frozen waste rock conditions below the ore body. With water being sprayed in a frozen environment, a resultant dense fog ensued and it was found that the original laser rangefinder cavity survey system was inadequate.

65 Some effort was made in an attempt to eliminate or reduce the fog, but this was unsuccessful.

An ultrasonic sensor was subsequently chosen as the replacement for the laser rangefinder due to its off-the-shelf availability, low cost, and, most importantly, the ability of the signal to penetrate the fog. During testing in the ore body, it was discovered that significant cavity wall erosion occurred above the jet as mining progressed downward and, as a result, it was determined that a final survey of the cavity (later called post cavity survey), prior to backfilling, would be required. Preferably, this survey would be completed as quickly as possible after jetting is complete, before any additional sloughing occurred. Since the ultrasonic sensor did not provide a high enough level of precision and resolution, the decision was made to again employ the use of a laser rangefinder. This post cavity survey was in addition to the ultrasonic survey, which was still completed between periods of jetting (interim cavity survey) to provide feedback to the operator. Through the process of field testing, it was found that the Cigar Lake 80 cavity shape could be roughly modelled by a cylinder of height ranging from 3 $\mathrm{m}$ to $15 \mathrm{~m}$ and a diameter ranging from $3 \mathrm{~m}$ to $5 \mathrm{~m}$.

In 2005, a review of current technology was conducted by the Saskatchewan Research Council (SRC), in which an MDL Cavity Auto-Scanning Laser System 
(C-ALS) [9] was identified at the most viable tool for the post cavity survey system [10]. It was small enough to inside the backfill pipe (5" inner diameter) and was equipped with a suitable deployment method and data processing software. In 2006, several ultrasonic range sensors were tested in a lab for the interim cavity survey system where it was found that the Senix ToughSonic TSPC-30S1 demonstrated the best performance for the Cigar Lake application [11.

Armed with a combination of the ultrasonic sensor and a laser scanning system, it would seem the bases would be covered; Senix ultrasonic to provide approximate range data to the operators during the jetting process (interim cavity survey) and the C-ALS to provide final, accurate, dimensional data for mine planning (post cavity survey). However, this paper questions whether it might be possible that a single solution could provide sufficiently accurate data during the jetting process. This would be ideal, having the potential to result in significant time and cost savings compared with two separate surveys. There exist emerging LiDAR systems which could have potential in cavity scanning applications (ex. CSIRO Zebedee [12]), though, with an investigation into other sensing technologies currently on the market, the SwissRanger ToF camera was identified as possessing sufficient characteristics to warrant an evaluation of its suitability for the Cigar Lake cavity survey application.

\subsection{Survey Sensor Evaluation}

Each of the three range finding devices uses the time-of-flight principle in which the time for a signal to travel from source to target, where it is reflected, and back is measured. The distance to the target can be calculated based on knowledge of signal speed. In order to systematically select the best sensor for the application, what follows is a brief review of each sensor's characteristics, as well as their advantages and disadvantages. See also Table 1.

An ultrasonic signal is a longitudinal, mechanical wave where the accuracy in range measurement relies on knowing the speed of sound in the particular medium. The geometry of the area surrounding the target and the angle of incidence to the target will also have an effect on the acquired measurements. It 
Table 1: Pre-study comparison of survey devices examined by this paper.

\begin{tabular}{lccc}
\hline & Senix ToughSonic & MDL MKIII & MESA SR4030 \\
& TSPC-30S1 & C-ALS & ToF Camera \\
\hline Survey Application & Interim Cavity & Post Cavity & Unknown \\
Cost & Low & High & Medium \\
Data Acquisition Rate & 20 points/s & 250 points/s & 50 frames/s \\
Grayscale Image & No & B\&W video & Yes \\
Packaging & IP68 & IP67 & IP67 \\
Fog/Water Conditions & Good & Poor & Unknown \\
Closed Space & Unknown & Good & Good \\
Surface Reflectivity & Unaffected & Unknown & Unknown \\
Range Accuracy & $0.2 \%$ of range & \pm 5 cm & \pm 15 mm \\
Development Stage & Custom & Commercial & Required \\
\hline
\end{tabular}

may be possible for the echo to be reflected away from the transducer and/or be reflected at multiple points before returning to the receiver. An ultrasonic signal has a relatively wide beam width and, if it were to strike an area composed of several distances, it would be impossible to resolve the smaller target component of the returned signal. The data acquisition rate for the Senix sensor is 20 points/s and a smoothed average of the range measurements is currently used for viewing data. This is expected to provide a reasonable amount of information for the JBS operators to make a decision as to whether certain areas require further jetting.

A laser device can emit a single light beam comprised of a high degree of coherence and very narrow width. Thus, it is possible to obtain very accurate 125 measurements from a laser with high angular resolution. However, it is also highly sensitive to particles (e.g., water droplets) within the medium, as well as the target's properties. In clear air, the attenuation is minimal but, for mining applications, where dust particulates and fog are often present, the attenuation 
can be severe [13]. Spurious readings may occur as photons are returned prior to the beam reaching its intended target. The target itself can also have an effect on the quality of the signal returned. On a smooth, shiny, or wet surface, specular reflection may occur and, depending on the angle of incidence, the reflected beam may not be returned to the receiver. There is also potential for absorption of the laser signal on the dark surfaces. The fog created within a cavity during jetting, as discovered during testing in 2000, will be a limiting factor in how soon the post cavity survey occurs once jetting is complete.

A time-of-flight (TOF) camera operates with the use of an infrared signal source. The MESA Imaging SwissRanger (SR4030) [14 ToF camera was chosen for the Cigar Lake application due to its small size, weight, and commercial availability. It uses a CCD/CMOS imaging sensor where the phase delay of the recovered signal is used to calculate target distance. From the single pixel values on the imaging sensor, a $176 \times 144$ pixel depth map is computed, totalling 25,344 range points per frame in a single image acquisition. Additionally, an amplitude image can be obtained from the offset of the signal providing a measure of the quality of the distance information [15]. Since the amplitude data can be viewed as a grayscale image, this characteristic offers visual representation of the target surface. Current reviews claim that it is foreseeable that ToF cameras will replace previous solutions, or alternatively complement other technologies, in many areas of application [16].

150

As shown in Table 1, the SwissRanger device is the fastest device, obtaining up to 50 frames of data per second, where the C-ALS obtains only 250 data points per second. The data from the laser is plotted as in real time but would take over a minute to collect and display what the MESA ToF can obtain in $1 / 50$-th of a second. With the dense point clouds obtained with the SwissRanger, it was proposed that it may be possible to filter spurious readings obtained in foggy conditions, and examine whether the resulting output could be interpreted. In this case, it would be possible to use the ToF camera sooner than the C-ALS in foggy conditions and potentially supplement or even replace the ultrasonic sensor. 
With regards to the mechanical robustness of the sensors, unlike the SwissRanger, laser systems generally employ several moving parts. Often with increased complexity, comes increased maintenance. It is known that in ideal conditions, laser systems have outperformed TOF cameras [17] for the purpose of very detailed 3D imaging, but for the Cigar Lake application, the TOF camera data could prove to be more reliable.

A distinct advantage of the C-ALS for a commercial application is that it is a complete off-the-shelf system, equipped with application software, integrated pitch and roll sensors, and a deployment method. To form a complete system with the ToF camera, further development, including adaptors, encoders, and software, along with required electronics and power, would be required. This study aims to evaluate whether the data acquired with the SwissRanger may offer advantages warranting further development for the Cigar Lake application.

\section{Laboratory and Field Data Collection}

This section describes the test environment, experiments, and field data collected to evaluate the range sensors considered for remote cavity surveying at the Cigar Lake mine.

\subsection{Experimental Test Cavity}

Experiments were conducted in a test cavity to demonstrate the capabilities and limitations of the devices proposed for use within the underground cavity application at Cigar Lake. These experiments could also provide a baseline for the interpretation of data obtained from any of the systems during mine production. Since the shape, size, and target reflectivity of the cavity have an effect on the sensor performance, the cavity testing space was designed to emulate the properties and size of a true cavity as closely as possible.

A hexagon-shaped wooden enclosure was built with wall-to-wall distances varying from $4.2 \mathrm{~m}$ to $5.0 \mathrm{~m}$. A wall shape similar to a cavity was constructed by manipulating diamond mesh. A stucco base was then applied to create a 
cavity-like surface. To finish, Cigar Lake core samples were examined and used as a guide to select colours for the stucco finish (see Figure 9(a). The uranium ore is very strong and likely to protrude further than the surrounding softer rock, after jetting is conducted. The high grade ore is also pitch black and, for this reason, the greatest protrusions on the test cavity walls were painted black (see Figure 2).

\subsection{Test Environments}

\subsubsection{Baseline}

Before beginning the series of various experiments, it was important to acquire a set of baseline data for observation of the C-ALS and SwissRanger ToF Camera performance in the 'ideal' cavity space. The C-ALS comes with a software package that allows for several options in the acquisition process . Figure 4 shows how the interval angle affects the visual detail of the 3D plots. There is an obvious improvement in the visual information provided between a scan taken with a $5^{\circ}$ interval to that of $1^{\circ}$ interval. However, the difference between the $1^{\circ}$ and $0.5^{\circ}$ intervals appears less obvious, even though there are approximately twice as many data points and the scan have taken twice as much time (see Figure 3). This will be a consideration for acquiring data in the field where time has a direct correlation with cost. At the mine, this data will be imported into Maptek $^{\mathrm{TM}}$ software, using I-Site ${ }^{\mathrm{TM}}$ for processing the scan data and Vulcan ${ }^{\mathrm{TM}}$ to access the cavity volume and compare against the mine plan.

Since the SwissRanger ToF Camera is not part of a complete scanning system, as the C-ALS is, a Celestron NexStar SE tripod was used to rotate the camera and output the angle of rotation. The ultrasonic sensor and SwissRanger were mounted to an adaptor designed by SRC, as shown in Figure 2

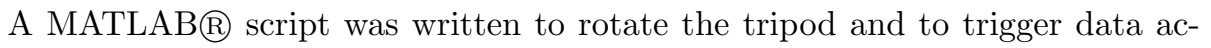
quisition from both sensors. The 3D Cartesian coordinate data $(x, y, z)$, along with a range, amplitude (grayscale), and confidence map (see Figure 5 from the SwissRanger and a specified number of range values from the Senix sensor were acquired at each angle interval. Four different data filtering modes were 


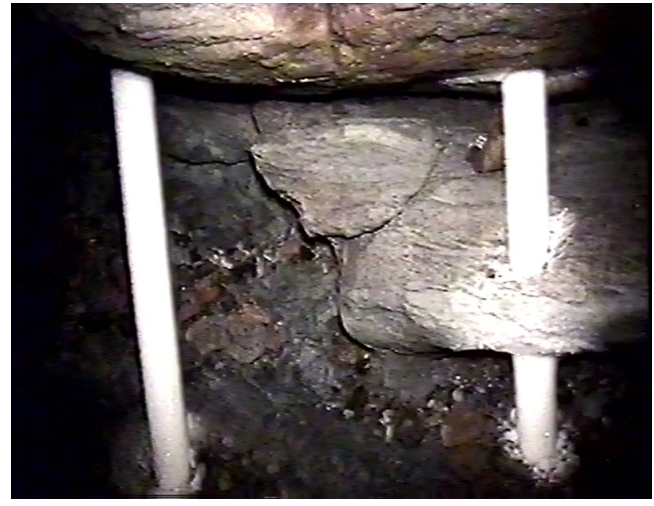

(a) A real Cigar Lake cavity with freeze pipes (image courtesy Cameco Corporation)

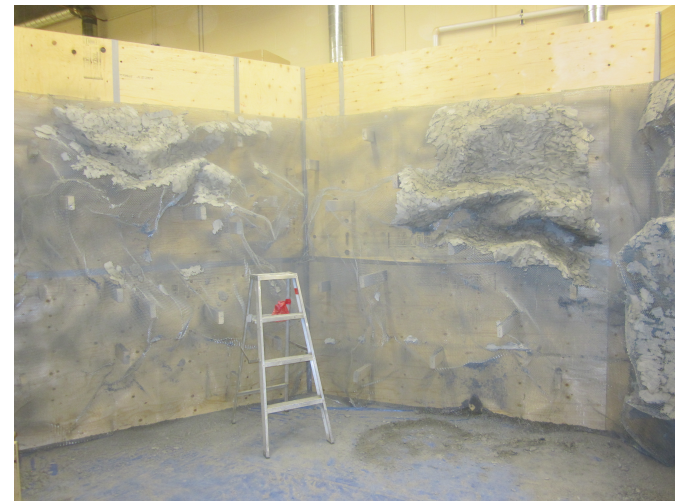

(b) Test cavity construction

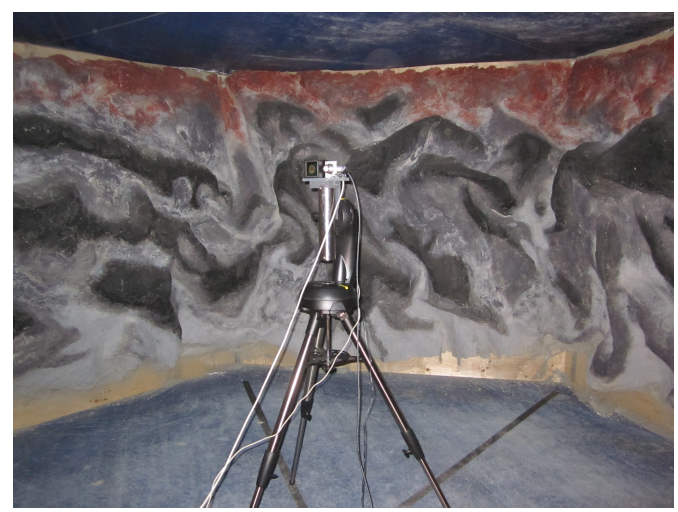

(c) Completed test cavity

Figure 2: Design and construction of test cavity 


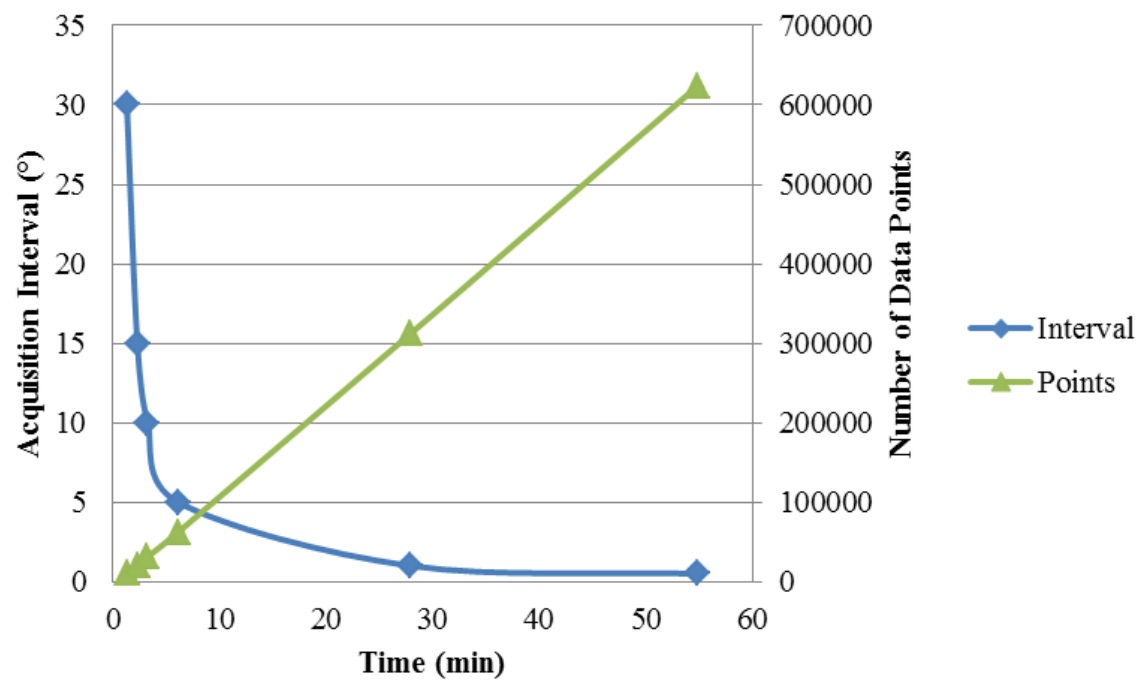

Figure 3: C-ALS baseline scan (vertical) demonstrating data acquisition time.

set on the SwissRanger during data acquisition. These included a Median Filter $(3 \times 3$ median filter run on the host PC), Neighborhood Filter $(5 \times 5$ hardware adaptive neighborhood filter), Median and Neighborhood Filter, and No Filters. The integration time, which is the length of time that the pixels are allowed to collect light, was also varied during data acquisition to observe the effect within the test cavity. In order to form a complete $3 \mathrm{D}$ point cloud from the $360^{\circ}$ scan, the data was saved in the point cloud library (PCL) . pcd format. PCL is a ${ }_{225}$ large scale, open project for 2D and 3D image and point cloud processing [18]. The camera viewpoint was obtained from the tripod encoder angle and recorded in the header of the PCD data file. The PCL viewer was then used to visualize the 3D point cloud data from the SwissRanger (see Figure 5).

\subsubsection{Water}

For the Cigar Lake application, the presence of water in the surveyed cavity is expected. During the process of jetting, the nozzle produces a jet of water that is directed upwards at an angle of $65^{\circ}$ from the axis of the sub. The inner 


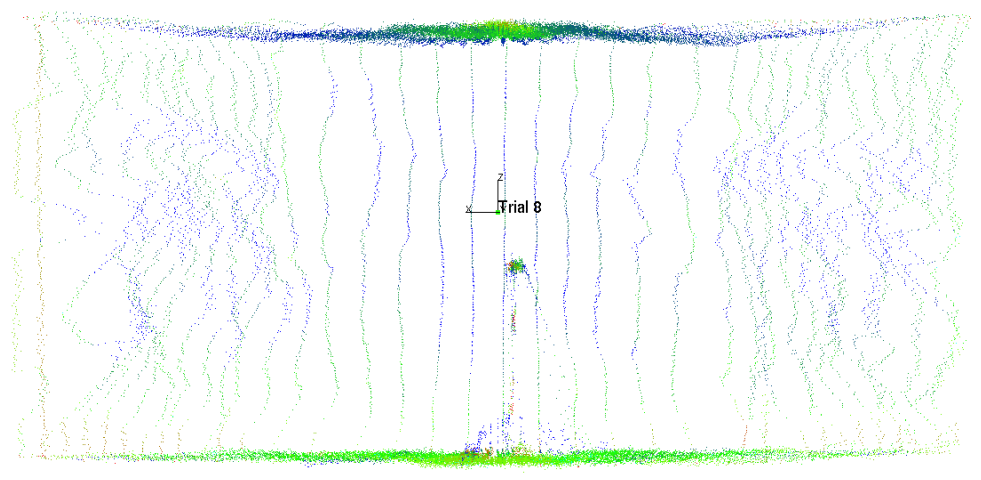

(a) $5^{\circ}$ interval

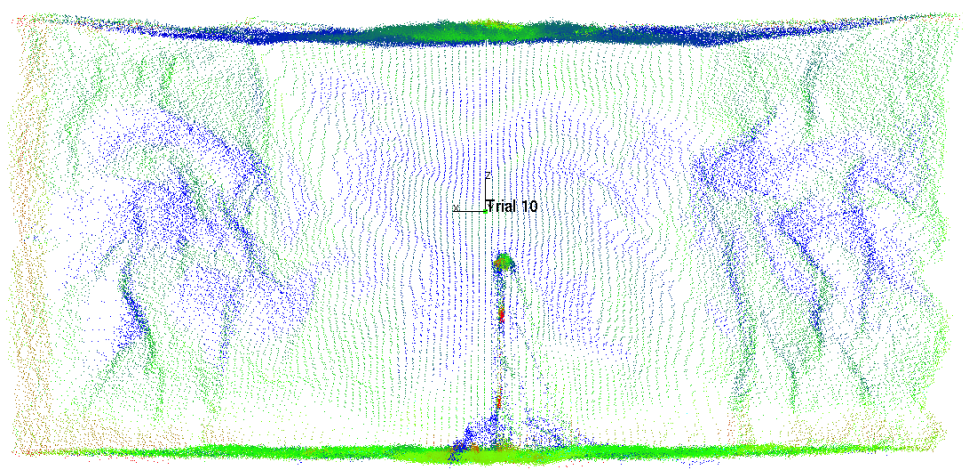

(b) $1^{\circ}$ interval

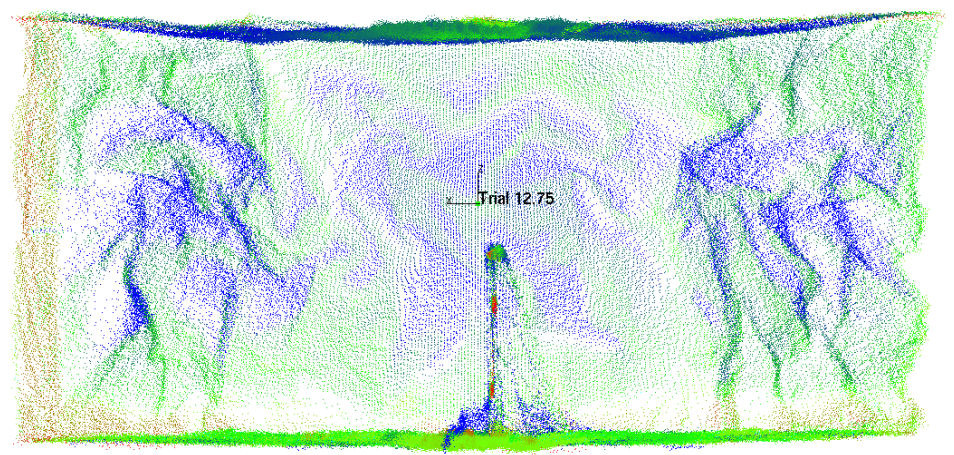

(c) $0.5^{\circ}$ interval

Figure 4: C-ALS vertical scan 3D plots shown with increasing acquisition intervals (colour scaled by signal strength with blue for low and red for high) 


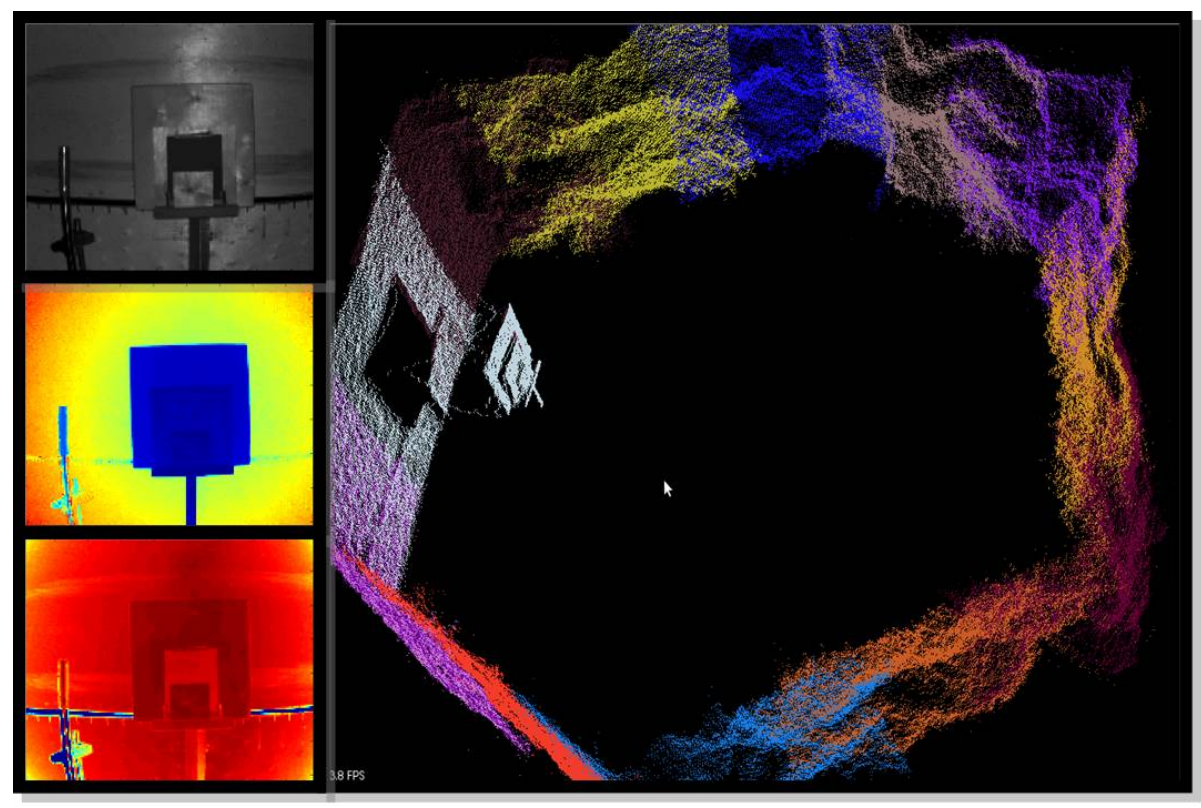

Figure 5: Left: Basic image acquisition from SwissRanger using MATLABß) (amplitude (grayscale) image, range image, and confidence map); Right: Point cloud data plotted using the PCL viewer 
pipe assembly supplies flow to the nozzle at pressures up to $100 \mathrm{MPa}$. The interim survey, using the Senix ultrasonic sensor, will give an indication of the cavity dimension during the process of jetting, but it is also expected that the surface will remain water saturated for the final, post cavity survey. Therefore, any sensor to be used must operate reliably with water on the target surface and potentially on the sensor itself.

In order to simulate the cavity environment after jetting, water was sprayed inside the test cavity, saturating the walls and roof and creating near $100 \% \mathrm{hu}-$ midity within the space. Data obtained from both the C-ALS and SwissRanger were compared to the baseline data and found to have no significant difference in the range data.

The water was also sprayed directly onto the sensor surfaces to observe what effect it would have on performance. For all sensors, the effect was most noticeable directly after spraying occurred because it caused a distortion of the signal. A shorter range (by $\approx 10 \mathrm{~cm}$ ) was measured with the Senix ultrasonic sensor and the data from the C-ALS appeared noisy with early signal returns. Noisy point cloud data was also observed with the SwissRanger and the look of a lens appeared on the amplitude image. The effect water had on each sensor was reduced in time due to heat dissipation from the sensors which caused the water to pool and evaporate.

\subsubsection{Freezepipes}

As part of the mining process at Cigar Lake, the ore and surrounding rock must be frozen prior to jetting. This is achieved through the installation of freeze pipes in a grid pattern through which brine is circulated to freeze the ground. It is known that jetting cavities will expose freeze pipes and it will be beneficial to know how the pipes will appear from each device, in order to identify them when surveying. If a freezepipe is identified, it could be taken into consideration when using the point cloud data to calculate the overall volume the cavity. Perhaps more importantly, a freeze pipe that has been exposed will need to be identified so it can be monitored when the brine is turned back on, 
to ensure no permanent damage has occurred.

The ultrasonic signal has a wide beam width and is unlikely to resolve a

freeze pipe and, furthermore, the current software uses a smoothing average, so it would be even more unlikely to distinguish a point reflected on a freezepipe from the adjacent cavity wall data. With the C-ALS, there are three typical scan interval settings. These are at $6^{\circ}, 3^{\circ}$, and $1^{\circ}$. It is perhaps obvious that with more data, you are more likely to easily detect the freeze pipes, but consideration of time must also be a factor. It was observed that with a vertical scan interval of $3^{\circ}$, the freeze pipe could be identified but without a high level of confidence.

In contrast, with the high density of range data obtained in a single image from the SwissRanger ToF camera and the corresponding amplitude image, the chance of a freezepipe going undetected would be highly unlikely. Object detection is clearly an advantage of the SwissRanger over the C-ALS, especially with consideration of the video frame rate data acquisition speed.

\subsubsection{Fog}

Due to the method of mining used at Cigar Lake, with water being sprayed in a frozen environment, the build up of fog is a significant issue for any survey sensor that is to be used in the jetted cavity. Approximate light, medium, and dense fogs were created with an Antariß fog machine for the purpose of testing the C-ALS and SwissRanger performance in the fog environment.

During the tests, various settings on both the SwissRanger and C-ALS were adjusted in effort to determine which might be best. The C-ALS possesses a 'Last-Hit' option that is suggested for use when there is water vapour or dust in the air. These obstructions in the air can cause the laser to reflect back to the instrument before reaching the intended target 19 . Thus, this option only processes the last portion of the signal returned, instead of the potentially erroneous early-returns. It was noted, however, that this option did not make an observable improvement on the data obtained within the test cavity. In comparing the baseline and dense fog data from the C-ALS, it could be seen that the point cloud obtained in fog still resembled the true surface but was 
scaled down, due to the early returns of signals on the fog. The new points appeared roughly $1 \mathrm{~m}$ from the true target surface. In application at the Cigar Lake mine, it will be important to know when there exists fog in the cavity, because such false early-returns may not be distinguishable from normal data and may cause significant errors in volume and production estimates.

When acquiring data from the SwissRanger, the four filtering modes (none, median, adaptive neighborhood, and both) and various integration times were

300 adaptive neighborhood filter was utilized and when the integration time was highest. It was observed that, although a correct amplitude image of the surface could be seen, the corresponding range point cloud was condensed according to the level of fog inside the test cavity (see Figure 6). The average range for the 305 Since the 2D image of the cavity wall still appeared to resemble the surface (see Figure 7), despite the small range values, it raised the question as to whether the accurate range values could be extracted or filtered with a lesser integration time, where part of the data still encompassed true range values.

In order to determine the possibility of extracting true range data from the SwissRanger in fog, the camera was set at two different positions - approximately $2 \mathrm{~m}$ and $4 \mathrm{~m}$ from the target surface within the test cavity - and data taken at several integration times, in the light, medium and dense fog conditions. As shown in Figure 8, at an integration time of 0 , the point cloud consists purely of 315 baseline (no fog) and medium fog conditions. At a higher integration time of 5.3 $\mathrm{ms}$, the baseline data still does not represent the true range, but it is possible to see the point clouds from the two different positions begin to separate. With the same integration time, but in fog, the point cloud begins being condensed for both positions. Finally, at the highest integration time of $25.3 \mathrm{~ms}$, the true average range is represented at approximately 2 and 4 meters, without fog in the cavity. With fog, it is exclusively early returns that are observed, thus demonstrating the difficulty in extracting the true range. 


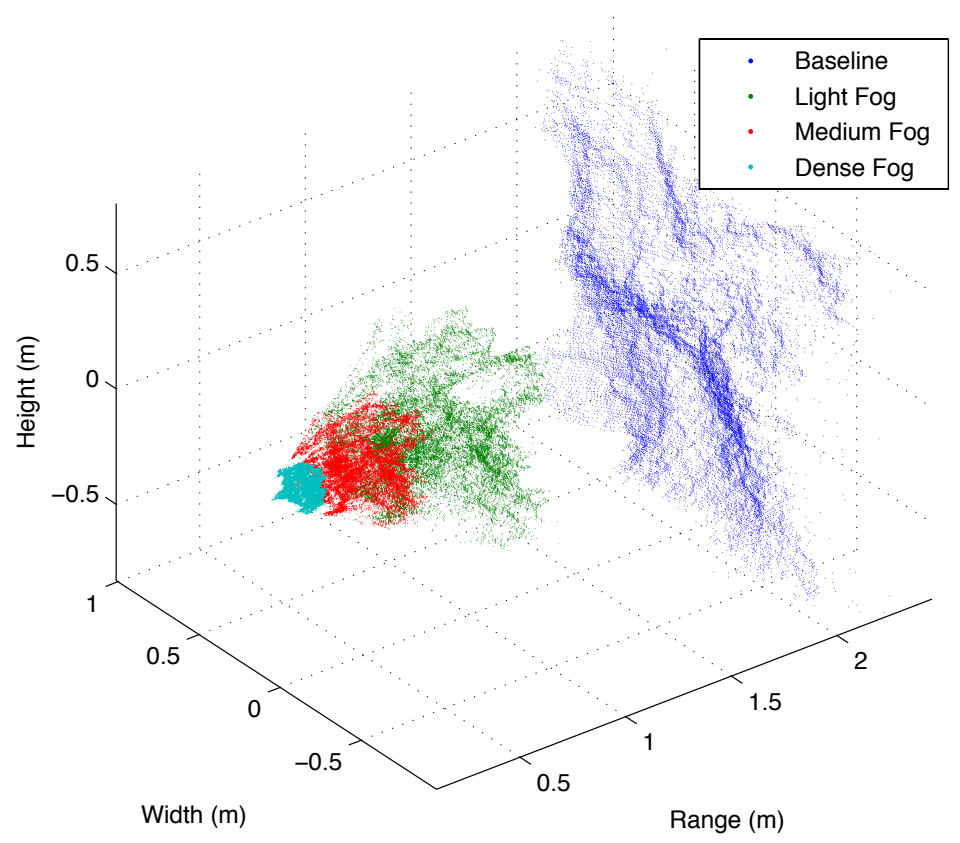

Figure 6: Point cloud data from SwissRanger in light, medium, and dense fog conditions inside the test cavity. The Baseline data set shows an approximate profile of the true cavity, while the impact of the fog can be seen by the remaining data sets. The camera is located at $(0,0,0)$. 


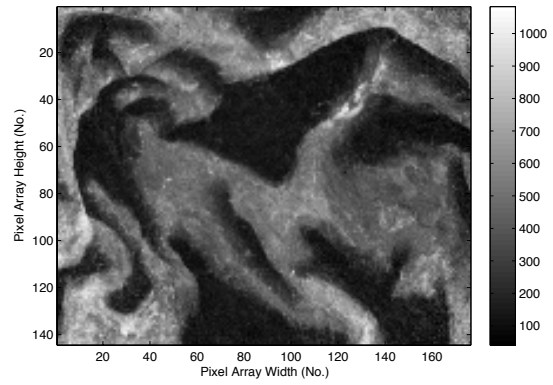

(a) Baseline Amplitude Image

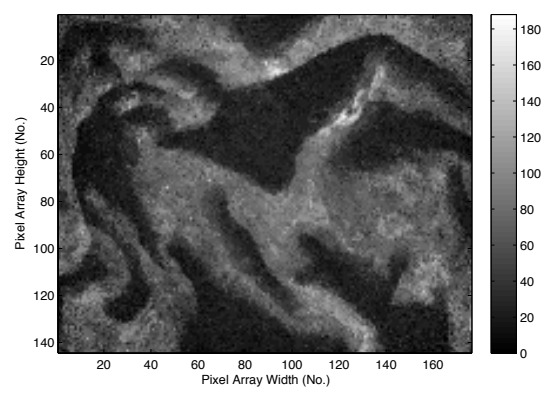

(b) Amplitude Image in Medium Fog



(c) Amplitude Image in Dense Fog

Figure 7: Amplitude Images from SwissRanger in Fog (Auto-scaled in MATLABß) 

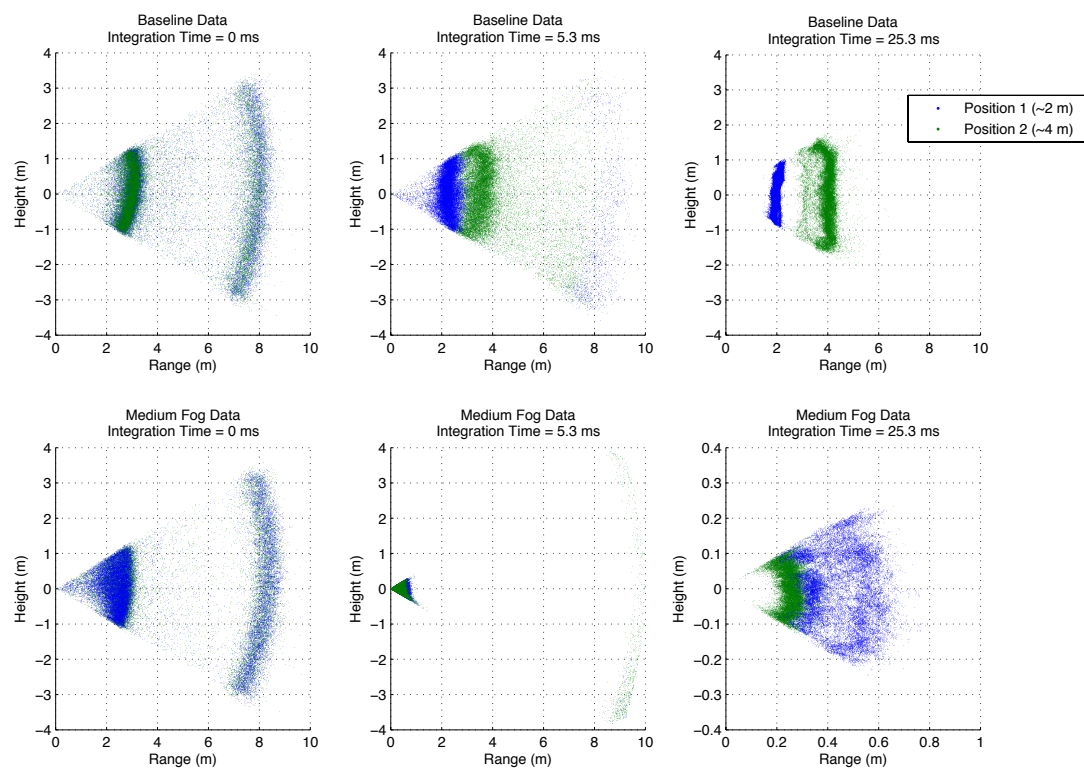

Figure 8: Side view of point cloud data from SwissRanger acquired from positions $2 \mathrm{~m}$ apart in no fog (top) and medium fog (bottom) conditions. Integration time is increasing from left to right. 
In the field, the fog particulates could be of a different size and composition automated segmentation could be explored (see Section 4.2).

The images acquired at Rabbit Lake, shown in Figure 10, demonstrate the potential of the SwissRanger for a short-range cavity survey device in a mine. The detail captured in the amplitude image could prove beneficial for remote visualization of the cavity by a human operator. The point cloud and range images demonstrate how the distance from the walls of a cavity could be shown with the data. Points that are beyond the max range of the camera are recorded with a value of zero. The confidence map reveals that 'bad' data could poten- 


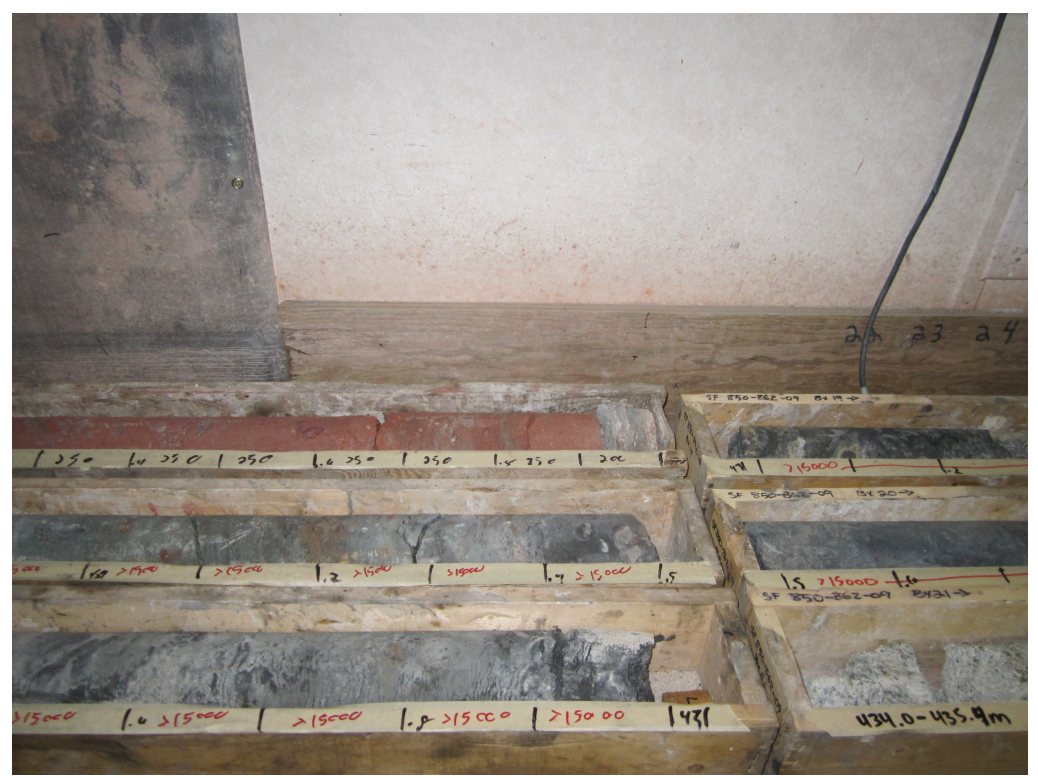

(a) Cigar Lake core sample

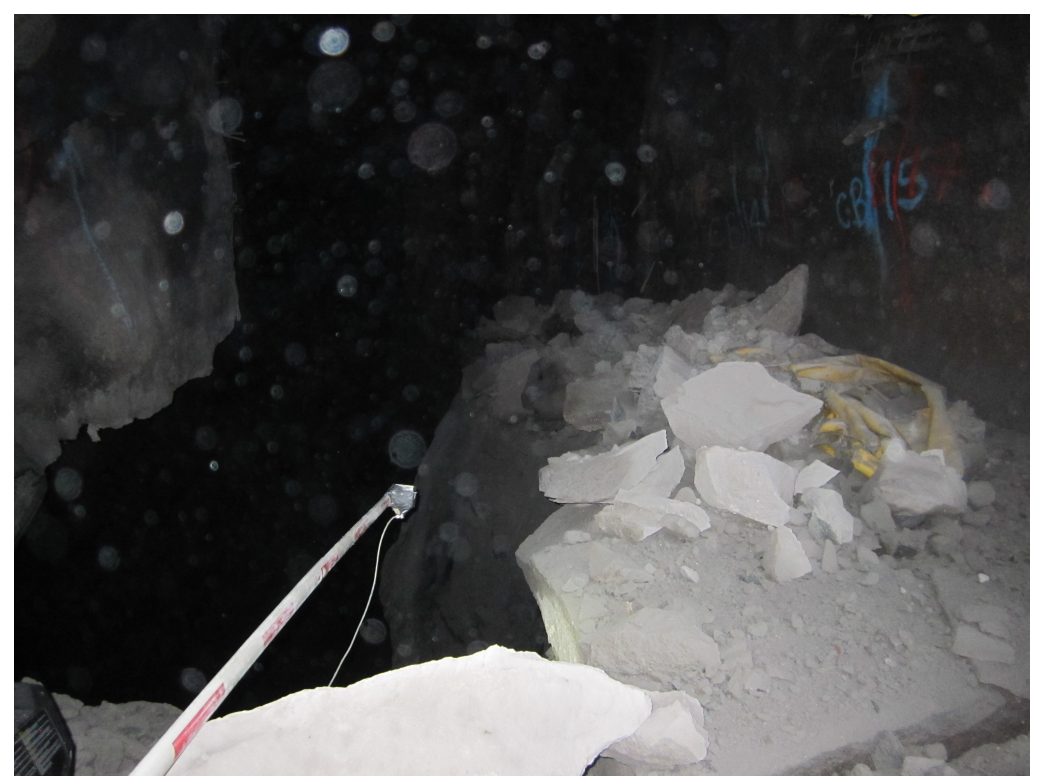

(b) Rabbit Lake stope

Figure 9: Field data acquisition with SwissRanger at Cigar Lake and Rabbit Lake 
tially be identified using this software tool since, for example, points beyond the range of the camera are blue (not confident). The images in Figure 10 indicate that the ToF camera would be effective in capturing accurate point cloud data from a rock face at close range, as might be encountered within a cavity.
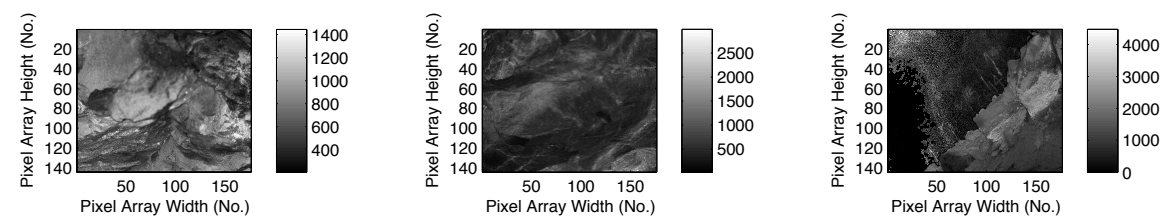

(a) Amplitude Images
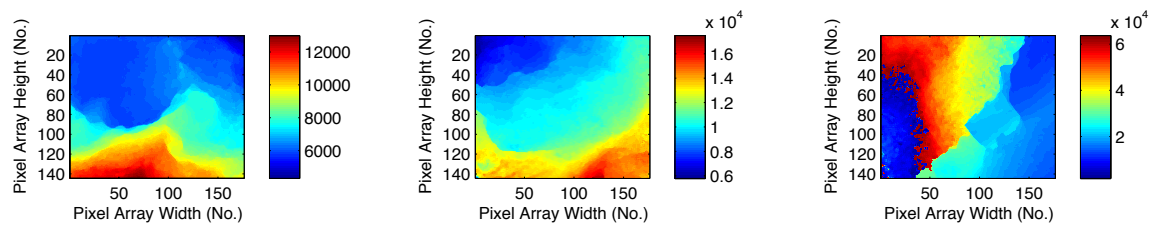

(b) Range Images
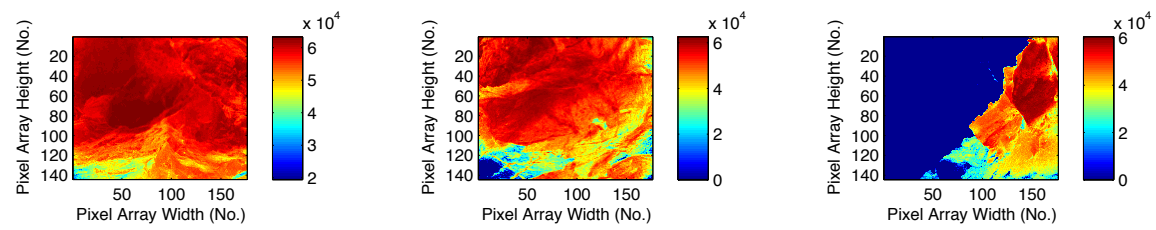

(c) Confidence Map

Figure 10: SwissRanger images acquired at an open stope at Rabbit Lake Mine

\section{Analysis}

This section describes some preliminary numerical analyses conducted on the data described in the previous section.

\subsection{Registration}

One advantageous characteristic of the ToF camera is the substantially large amount of data that can be acquired in a very short amount of time, as it opens 
multiple options for analysis. An ICP registration algorithm could be applied since it is generated by using a combination of distance and amplitude measurements and their temporal variations [21] and could suggest a strong starting point for matching. Further filtering of data (ex. pass through filter, down 
sampling, statistical outlier removal) to create a cleaner data set or an exploration of different feature extraction techniques to identify rock features in the cavity (ex. Normal Estimation [22, Point Feature Histograms [23, 3]) could potentially improve results.

With regards to the Cigar Lake remote cavity application, the most practical use of registration might be to use it to supplement or provide correction to data acquired with a position encoder. As suggested by Hedlund [24, a good solution to improving registration is to attach odometry equipment to the ToF camera being used. The encoder would provide an initial position estimate to the registration algorithm. For future work, it may be a useful exercise to introduce various levels of error to the initial pose estimate and observe the success in using registration to correct the viewpoint angle of the camera.

\subsection{Segmentation}

Segmentation is the process by which an algorithm segments a point cloud into distinct clusters according to certain parameters. Since there was success in obtaining amplitude images that visually represented the surface of the test cavity, even in the dense fog environment (see Figure $7(\mathrm{c})$, the question arose as to whether the returned signal amplitude could be used to identify the extents of the ore body, or to differentiate between minerals.

As a simple initial test, a pass through filter was used to remove intensity data which fell between a range of values, and was found to be successful (see Figure 11(b) For example, it was shown that the signals which had been returned from the portion of the test cavity wall which was painted black (representative of uranium pitchblend), could be filtered. There exist far more sophisticated algorithms, for use with data acquired with 3D imaging devices,

which attempt to improve robustness to texture, scale, and viewpoint. Because the amplitude of the returned signal was observed to be distinct enough to manually filter according to a range of values, the PCL Conditional Euclidian Clustering was tested to see if it could automatically perform a similar task. This segmentation algorithm clusters points based on Euclidean distance and a 
user-customizable condition (i.e., distance in amplitude space) [25]. Successful clustering was observed when the defined set condition required the difference between amplitudes to be less than a value of one (see Figure 11). The SwissRanger amplitude measurement 21] is without standard units, but is proportional to the square of the measured distance, scaled to equal one at a distance ${ }^{430}$ of half of the full phase distance.

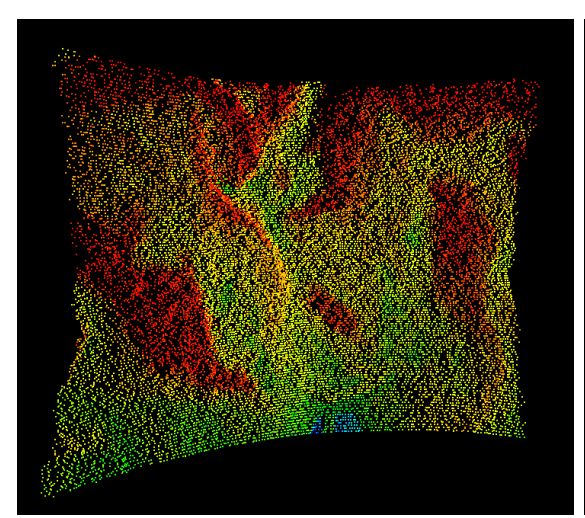

(a) Intensity point cloud

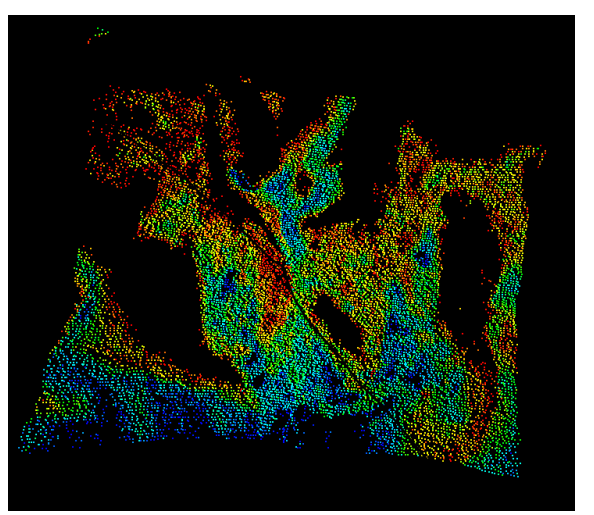

(b) Pass through filter

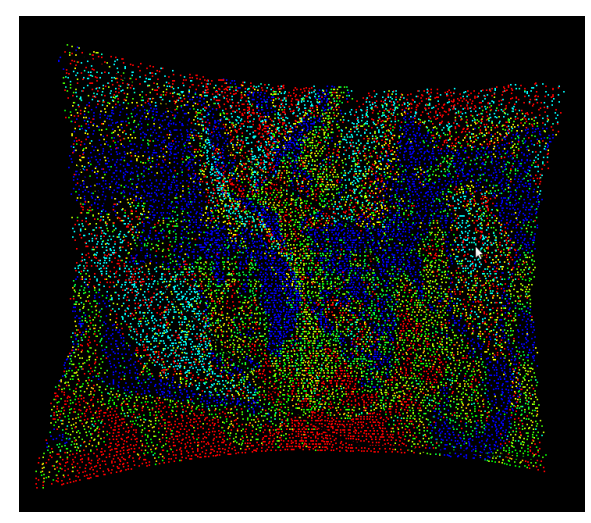

(c) Conditional Euclidean clustering

Figure 11: Segmentation applied to intensity point cloud 


\section{Discussion}

The purpose of this study was to evaluate the use of a ToF camera in a remote cavity surveying application, with comparison to devices planned for use at the Cigar Lake mine site. Potential advantages included data acquisition and range are very low.

The high point cloud density obtained with the SwissRanger also offered a distinct advantage over the other devices tested. This was demonstrated rate, point cloud density, and cost.

It was clear, as shown in Table 1, that the ToF camera (SwissRanger) had the fastest data acquisition rate. The video frame rate of 50 frames/s would provide a distinct advantage for operators to visualize the inside of the cavity, especially with the grayscale (amplitude) images. Comparatively, the C-ALS is equipped with a separate video camera component which is mounted at the end of the probe, but is only intended for use during deployment, since its resolution through the use of representative environments inside the test cavity. It was found that the moisture or water on the cavity walls had insignificant effects on the data obtained from both the C-ALS and the SwissRanger. However, when water was sprayed on each lens' surface, both the C-ALS and the SwissRanger output noisy data due to early signal returns, but with the SwissRanger, the distortion could be identified and diagnosed. The freeze pipes in the test cavity also showed that objects at a distance could be more easily identified from a device with high point cloud density. It was possible to obtain enough data points with the C-ALS in order to positively identify a freeze pipe, but the SwissRanger would be capable of achieving this in a fraction of the time, and with less ambiguity.

Inside the fog environment, it was found that the point cloud density of the SwissRanger did not offer an advantage. The various filter modes and integration times were tested, with results showing that the true range data could not be simply extracted. It was already known that the C-ALS would not provide reliable data in fog from previous testing with a laser range finder 
at Cigar Lake, and this was reconfirmed in the test cavity. Of the two signals, however, the C-ALS was able to penetrate further into the fog (N.b., the C-ALS emits a higher powered signal), though it remains that it should not be relied upon to provide range data in fog conditions. As expected, the ultrasonic sensor was the only sensor to provide true range data in the fog.

One interesting observation noted during the fog testing was the accurate representation of the target's surface that appeared in the amplitude image, even in the dense fog conditions. This lead to a review of sensors on the market that claim to have obscurant penetrating capabilities, in an attempt to determine what characteristics of the device made this possible [26, 27, 28, 29]. Additionally, an investigation into whether an infrared device might be able to provide depth information within the fog conditions was carried out. In order to 'see-through' the fog, it is necessary to have advanced filtering techniques that are integrated into both the hardware and software, of devices that would be similar to the SwissRanger [30. If it were possible to examine the analog pulses received by the device, there could be the potential for identifying those that have returned from the target surface, instead of being returned by the fog 31 .

In examining IR devices for stereo vision, it was found that some promising success had been found when using thermal IR in the long wavelength infrared spectrum [32, 33]. In this spectrum, one needs only to examine the IR emission from surfaces, rather than contend with the added complication of the reflected signals. Since the SwissRanger is a near-infrared device, without potential to examine received signals directly, it was determined that using this device, even in a stereo setup, would not be likely to reveal any added information about the range of a target in fog.

From a business perspective, the cost of an entire survey system is also a consideration. Factors may include the sensor cost, reliability or maintenance requirements, time savings from survey completion time, and value in informa490 tion gained. It was found that the interim survey (in fog) could not be completed with the SwissRanger, implying that its use at Cigar Lake would be for the post 
cavity survey (as the C-ALS). Studying the reliability and maintenance aspects for each sensor was outside the scope of this study. The C-ALS is already developed as a complete survey system and adapters have already been specially designed for the deployment method [34]. For the post cavity survey, the SwissRanger certainly has potential to collect a full survey of the cavity in a shorter time period than the C-ALS, though software would need to be developed to fully evaluate a difference processing time. Promise has also been shown in potential increased value from the dense point cloud and amplitude images from the SwissRanger, which allow for advanced data processing techniques such as registration and segmentation.

As discussed in section 4.2 , potential for segmenting the images according to mineral type has been shown and may prove to be of particular use in visualizing the cavity and identifying the limits of the ore body. Another option for strong consideration in segmentation is by use of the rotation-invariant descriptor, intensity-domain spin images [35]. This algorithm uses a two-dimensional histogram encoding the distribution of image brightness values in the neighborhood of a particular reference (centre) point. Future work may include the incorporation of intensity-domain spin image descriptors into an algorithm which will segment the test cavity appropriately. Ideally, these algorithms would also be tested in the field, but the unavailability of a cavity containing uranium ore, and the inability to validate the success of segmentation, currently makes it not possible. The use of a registration algorithm may also aid in position correction of data obtained from an encoder. Both of these options are not possible with the C-ALS

\section{Conclusions}

With the construction of a test cavity, and by simulating the environments that are expected to be present in underground cavities constructed with a jet boring system, it was possible to evaluate the data that could be obtained by 
environment with freeze pipes and with water, the quantity and high speed of data acquisition would be an advantage in using the ToF camera. Furthermore, the amplitude image, which appears as a grayscale photograph, would provide an additional mode of visualization for JBS operators who would be making decisions based on the data obtained. In the fog, as expected, it was shown that neither the C-ALS nor the SwissRanger could be used to obtain reliable range data. Other devices that have hardware and software claiming to filter out early returns from fog or dust are commercially available, but not tested or developed for this application.

Data obtained at Cigar Lake showed the variation in amplitude data for the various mineral compositions in a core sample, and data from Rabbit Lake showed how the range and amplitude images would appear when taken from an underground rock face. The field data provided confirmation that a ToF camera could be used for a post-cavity survey, just as the C-ALS, after jetting ${ }_{535}$ is complete. There is also the possibility that the SwissRanger amplitude data could be used to identify the extents of the ore body with segmentation, and that registration might be used to further correct pose information obtained from an encoder.

The C-ALS had been chosen for post cavity scanning at Cigar Lake because 540 it is a readily available and complete system that incorporates deployment, pitch and roll sensors, and application-ready software. Further field testing, data analysis, and hardware and software development would be required to integrate a ToF camera into an automated system for surveying remote cavities. Depending on long-term costs sustained with use of the C-ALS, the investment in developing a ToF camera survey tool for acquiring data in a cavity environment, and the additional information it may provide, could prove of value.

\section{Acknowledgements}

This research was funded in part by the Natural Sciences and Engineering Research Council of Canada (NSERC) and Cameco Corporation. The 

with experiments.

\section{References}

a [1] C. S. Bishop, G. J. H. Goddard, A. G. Mainville, E. Paulsen, Cigar Lake Project: National Instrument 43-101 Technical Report, Tech. rep., Cameco Corporation, Saskatoon, SK (2012).

URL http://www.cameco.com/common/pdf/mining/Cameco_-_Cigar_ Lake_Technical_Report.pdf

[2] P. J. Besl, N. D. McKay, A Method for Registration of 3-D Shapes, IEEE Transactions on Pattern Analysis and Machine Intelligence 14 (2) (1992) $239-256$.

п [3] R. B. Rusu, Semantic 3D Object Maps for Everyday Manipulation in Human Living Environments, KI - Künstliche Intelligenz 24 (4) (2010) 345348. doi:10.1007/s13218-010-0059-6. URL http://link.springer .com/10.1007/s13218-010-0059-6

[4] S. Rusinkiewicz, M. Levoy, Efficient variants of the ICP algorithm, in: Proceedings Third International Conference on 3-D Digital Imaging and Modeling, IEEE Comput. Soc, Quebec City, 2001, pp. 145-152. doi:10.1109/IM.2001.924423.

[5] Z. Zhang, Iterative Point Matching for Registration of Free-Form Curves, Tech. rep., Institut National de Recherche en Informatique et en Automatique (1992).

[6] A. Hoover, G. Jean-baptiste, X. Jiang, P. J. Flynn, H. Bunke, D. B. Goldgof, K. Bowyer, D. W. Eggert, A. Fitzgibbon, R. B. Fisher, An Experimental Comparison of Range Image Segmentation Algorithms, IEEE transactions on pattern analysis and machine intelligence 18 (7) (1996) 673-689. 
[7] T. Rabbani, F. A. van den Heuvel, G. Vosselman, Segmentation of point clouds using smoothness constraint, in: ISPRS Commission V Symposium 'Image Engineering and Vision Metrology', Dresden, 2006, pp. 248-253.

[8] M. Wacker, 2000 Jet Boring Systems Test: Final Report, Tech. rep., Cigar Lake Mining Corporation, Saskatoon, SK (2001).

[9] MDL Website.

URL http://www.mdl-laser.com

[10] C. Murray, K. Young, T. Kaminski, B. Lung, K. Babich, B. Smith, M. Paulson, K. Wickenhauser, Review of the Cavity Survey System, Post Survey Tool and Casing Hole Survey System, Tech. Rep. 11265, Saskatchewan Research Council, Saskatoon (2005).

[11] N. Peter, T. Kaminski, Q. Wan, K. Young, CVSS Ultrasonic Sensor Report, Tech. rep., Saskatchewan Research Council, Saskatoon (2006).

[12] M. Bosse, R. Zlot, P. Flick, Zebedee: Design of a Spring-Mounted 3-D Range Sensor with Application to Mobile Mapping, IEEE Transactions on Robotics 28 (5) (2012) 1104-1119. doi:10.1109/TR0.2012.2200990.

1 URL http://ieeexplore.ieee.org/lpdocs/epic03/wrapper.htm? arnumber $=6220900$

[13] G. Brooker, R. Hennessey, C. Lobsey, M. Bishop, R. St, J. Building,

口 E. Widzyk-capehart, Seeing through Dust and Water Vapor : Millimeter Wave Radar Sensors for Mining Applications, Journal of Field Robotics 24 (7) (2007) 527-557. doi:10.1002/rob URL www . interscience.wiley.com

[14] MESA Imaging Website. URL http://mesa-imaging . ch

[15] T. Oggier, R. Kaufmann, M. Lehmann, P. Metzler, G. Lang, M. Schweizer, M. Richter, B. Büttgen, N. Blanc, K. Griesbach, U. B, K.-H. Stegemann, 
C. Ellmers, 3D-Imaging in Real-Time with Miniaturized Optical Range Camera, Tech. rep., Swiss Center for Electronics and Microtechnology, Zuerich, Switzerland (2004).

口 URL http://mesa-imaging.ch/dlm.php?fname=pdf/opto_4_1_v1_0. $\mathrm{pdf}$

${ }_{610}[16]$ S. Foix, G. Alenya, C. Torras, Lock-in Time-of-Flight (ToF) Cameras: A 1. Survey, IEEE Sensors Journal 11 (9) (2011) 1917-1926. doi:10.1109/ JSEN.2010.2101060

a URL http://ieeexplore.iee.org/stamp/stamp.jsp?tp=\&arnumber= 5686908\&isnumber $=5961208$

${ }_{615}[17]$ Y. Cui, S. Schuon, D. Chan, S. Thrun, C. Theobalt, 3D shape scanning with a time-of-flight camera, in: 2010 IEEE Computer Society Conference on Computer Vision and Pattern Recognition, IEEE, 2010, pp. 1173-1180. doi:10.1109/CVPR.2010.5540082.

口 URL http://ieeexplore.ieee.org/lpdocs/epic03/wrapper.htm? arnumber $=5540082$

[18] Point Cloud Library (2013).

URL http://pointclouds.org/

[19] Measurement Devices Limited, C-ALS MANUAL Issue 5.01, Tech. Rep. 5 (2011).

625 URL WWw.mdl-laser.com

[20] G. C. Sharp, S. W. Lee, D. K. Wehe, ICP Registration Using Invariant Features, IEEE transactions on pattern analysis and machine intelligence 24 (1) (2002) 90-102.

[21] Anonymous, SR4000/4500 User Manual Version 3.0, Tech. rep., MESA ${ }_{630}$ Imaging AG, Zurich, Switzerland (2013).

a URL http://www.mesa-imaging.ch/dlm.php?fname=customer/ Customer_CD/SR4000_SR4500_Manual.pdf 
[22] K. Klasing, D. Althoff, D. Wollherr, M. Buss, Comparison of Surface Normal Estimation Methods for Range Sensing Applications, in: 2009 IEEE International Conference on Robotics and Automation, IEEE, Kobe, Japan, 2009, pp. 3206-3211. doi:10.1109/ROBOT.2009.5152493. URL http://ieeexplore.ieee.org/lpdocs/epic03/wrapper.htm? arnumber $=5152493$

[23] R. B. Rusu, N. Blodow, M. Beetz, Fast Point Feature Histograms (FPFH) for 3D Registration, in: 2009 IEEE International Conference on Robotics and Automation, IEEE, Kobe, Japan, 2009, pp. 3212-3217. doi:10.1109/ROBOT.2009.5152473 URL http://ieeexplore.ieee.org/lpdocs/epic03/wrapper.htm? arnumber $=5152473$

[24] T. Hedlund, Registration of multiple ToF camera point clouds, Master's thesis, UmeåUniversity (2010).

口 URL http://www.diva-portal.org/smash/get/diva2:327071/ FULLTEXT01.pdf

[25] PCL, Conditional Euclidean Clustering. $\mathrm{UR}$ http://pointclouds.org/documentation/tutorials/ conditional_euclidean_clustering.php

[26] E. Trickey, P. Church, X. Cao, Characterization of the OPAL obscurant 1 penetrating LiDAR in various degraded visual environments (2013). doi: $10.1117 / 12.2015259$ URL http://dx.doi.org/10.1117/12.2015259

[27] Anonymous, Seeing Through Fog and rain with a Thermal Imaging Camera, Tech. rep., FLIR Commercial Vision Systems (2012).

URL www.flir.com

[28] S. Vidas, S. Sridharan, Hand-held Monocular SLAM in Thermal-infrared, 660 in: 2012 12th International Conference on Control, Automation, Robotics \& Vision, Vol. 2012, 2012, pp. 859-864. 
[29] C. Brunner, T. Peynot, T. Vidal-Calleja, J. Underwood, Selective Combination of Visual and Thermal Imaging for Resilient Localization in Adverse Conditions : Day and Night, Smoke and Fire, Journal of Field Robotics 30 (4) (2013) 641-666. doi:10.1002/rob

[30] B. Short, 3D Flash LADAR Helicopter Landing Sensor for Brownout and Reduced Visual Cue.

URL ASC3D.com

[31] X. Zhu, P. Church, M. Labrie, LIDAR for obstacle detection during helicopter landing, in: M. D. Turner, G. W. Kamerman (Eds.), Proc. SPIE 6950, Laser Radar Technology and Applications XIII, 69500T, Vol. 6950, 2008, pp. 69500T-69500T-8. doi:10.1117/12.777160 URL http://proceedings.spiedigitallibrary.org/proceeding. aspx?articleid $=835301$

[32] K. Hajebi, Structure from Infrared Stereo Images, Ph.D. thesis, University of Waterloo (2007).

[33] A. Rankin, A. Huertas, L. Matthies, M. Bajracharya, C. Assad,

n S. Brennan, P. Bellutta, G. W. Sherwin, Unmanned ground vehicle perception using thermal infrared cameras 8045 (2011) 804503-804503-26. doi:10.1117/12.884349. URL http://proceedings.spiedigitallibrary.org/proceeding. aspx?articleid=1350998

[34] General Machine Products Co., Cameco Corporation, Cameco Pushes New Borehole Survey Method, Engineering and Mining Journal (November) (2012) 62-64.

URL http://emj . epubxp.com/i/94438

[35] S. Lazebnik, C. Schmid, J. Ponce, A sparse texture representation using local affine regions., IEEE transactions on pattern analysis and machine intelligence 27 (8) (2005) 1265-78. doi:10.1109/TPAMI.2005.151. 